\title{
Immunotherapy for the Post-Infectious Sequela of SARS-CoV-2 Infection
}

\author{
David S. Younger \\ School of Medicine, City University of New York, New York, NY, USA \\ Email: youngd01@nyu.edu
}

How to cite this paper: Younger, D.S. (2020) Immunotherapy for the Post-Infectious Sequela of SARS-CoV-2 Infection. World Journal of Neuroscience, 10, 117-120.

https://doi.org/10.4236/wjns.2020.102013

Received: May 7, 2020

Accepted: May 15, 2020

Published: May 18, 2020

Copyright (c) 2020 by author(s) and Scientific Research Publishing Inc. This work is licensed under the Creative Commons Attribution International License (CC BY 4.0).

http://creativecommons.org/licenses/by/4.0/

\begin{abstract}
The SARS-CoV-2 2019 pandemic is creating challenges to the management of post-infectious autoimmunity in childhood and adult Covid-19 cases due to its high case fatality. Nearly all of the agents envisioned to treat Covid-19 illness, including the newly recognized pediatric multi-system inflammatory syndrome, impact post-infectious mechanisms in keeping with the multiplier effect of infection, immunity and inflammation, known as I-Cubed $\left(\mathrm{I}^{3}\right)$.
\end{abstract}

\section{Keywords}

SARS-CoV-2, Covid-19, Intravenous Immune Globulin, IVIg, Pediatric Multi-System Inflammatory Syndrome

\section{(c) (i) Open Access}

The SARS-CoV-2 ([Covid-19.1]) pandemic is proving to be associated with high-case fatalities in both children and adults due to a dysregulated, post-infectious autoimmunity response, analogous to the cytokine storm of severe viral influenza illness [1]. The potentially devastating outcome of uncontrolled post-infectious autoimmunity due to SARS-CoV-2 exposure is more severe and long lasting than the infection itself especially in vulnerable patients, including young children with multi-system inflammatory syndrome (New York State Department of Health, Bureau of Communicable Disease Control. Health Advisory: Pediatric multi-system inflammatory syndrome potentially associated with coronavirus disease (COVID-19) in children. May 6, 2020) and older adults with acute respiratory distress syndrome [2]. Recognizing the importance of a given patient's immune response to the SARS-Cov-2 exposure, subjects are now being recruited to participate in studies to examine B- and T-cell repertoire and immune responses during the acute and resolved phases of Covid-19 infection at home and in the hospital (ClinicalTrials.gov Identifier: NCT04362865). 
Four immunotherapeutic approaches are being used to stem the Covid-19 pandemic by targeting the immune system, in keeping with the multiplier effect of infection, immunity and inflammation known as I-Cubed $\left(\mathrm{I}^{3}\right)$ [3]. The oral antimalarial drug hydroxychloroquine was the first highly publicized agent recognized for its immune-mediated mechanisms of chemotaxis, phagocytosis and superoxide production by neutrophils to inhibit SARS in vitro [4]. It was administered in an open-label non-randomized clinical trial of 20 adults and minors with severe Covid-19 illness with improvement, and later made widely available as prophylaxis [5]. An observational study of 1446 hospitalized adult patients at a New York City hospital with Covid-19 illness did not show a significant association between hydroxychloroquine use and intubation or death (hazard ratio, 1.04, $95 \%$ confidence interval, 0.82 to 1.32 ), with similar findings in multiple sensitivity analyses [6]. Randomized, controlled trials of hydroxychloroquine in patients with Covid-19 are needed to establish efficacy. A phase IIB study to evaluate the efficacy of hydroxychloroquine and azithromycin to prevent hospitalization and death in symptomatic adult outpatients with Covid-19 caused by SARS-CoV-2 infection has not started recruiting subjects (ClinicalTrials.gov Identifier: NCT04358068).

The biological agent remdesivir, a nucleotide analogue prodrug that inhibits viral RNA polymerases that showed in vitro activity against SARS-CoV-2 compassionately treated a cohort of 61 adults patients with improvement in two-thirds [7]. A clinical protocol allowing expanded access to remdesivir (ClinicalTrials.gov Identifier: NCT04323761), and several clinical studies have begun recruiting subjects in an randomized, open-label, controlled clinical trial, in collaboration with the World Health Organization (WHO) (ClinicalTrials.gov Identifier: NCT04330690). A phase III randomized study comparing the safety and efficacy and antiviral activity of two remdesivir regimens with respect to clinical status is recruiting subjects (ClinicalTrials.gov Identifier: NCT04292899). An open label study to evaluate the safety and antiviral activity of remdesivir with moderate Covid-19 compared to standard of care treatment is recruiting subjects (ClinicalTrials.gov Identifier: NCT04292730).

Convalescent plasma transfusion of SARS-CoV-2-specific IgG and neutralizing antibodies have been administered in uncontrolled case series to critically ill adult patients with Covid-19 with clinical improvement [8]. These preliminary findings suggest a role for transfusion therapy in the treatment of critically ill patients with COVID-19). A pilot prospective study collecting plasma to measure neutralizing antibodies to SARS-Cov-2 in recovered subjects has not yet starting recruiting participants (ClinicalTrials.gov Identifier: NCT04344977).

In retrospect, treatment with 2 grams per kilogram high-dose IVIg therapy administered to 3 adult patients over 4-5 consecutive days in the early stages of clinically apparent SARS-Cov-2 viremia, alone (1 patient) or in association with antiviral and antibacterial antibiotics showed clinical stabilization and were uneventfully discharged from the hospital [9]. Early administration of IVIg is 
first-line therapy in children with Kawasaki Disease (KD) that appears to be missed or delayed during the Covid-19 pandemic [10], but may also be etiologically related to SARS-CoV-2 infection. A single-center, randomized, open-label, controlled study in Peking China is evaluating the safety of IVIg in conjunction with standard care for severe 2019-nCov pneumonia has not started recruiting subjects (ClinicalTrials.gov identifier NCT04261426). However, no similarly available studies have been announced in the US.

An anecdotal prospective analysis of 55 children and adults treated with maintenance (400 $\mathrm{mg} / \mathrm{kg}$ monthly) and high-dose ( 2 grams $/ \mathrm{kg}$ ) IVIg therapy to treat diverse acquired and post-infectious autoimmune neurological disorders, found no new cases of SARS-CoV-2 stratified by a single home infusion service via phone interviews at the height of the Covid-19 pandemic (when it would have been impermissible for a nurse to enter the home) [11]. This uncontrolled observation suggests that IVIg therapy may yet have an important role in Covid-19 illness prevention among vulnerable individuals. However, a longer period of follow-up of this cohort will be necessary to confirm these observations, as are further controlled studies to identify the dose and frequency of IVIg treatment to confer prophylactic efficacy.

\section{Conflicts of Interest}

The author declares no conflicts of interest regarding the publication of this paper.

\section{References}

[1] Guo, X.J. and Thomas, P.G. (2017) New Fronts Emerge in the Influenza Cytokine Storm. Seminars in Immunopathology, 39, 541-550. https://doi.org/10.1007/s00281-017-0636-y

[2] Guan, W.J., Ni, Z.Y., Hu, Y., et al. (2020) Clinical Characteristics of Coronavirus Disease 2019 in China. The New England Journal of Medicine, 382, 1708-1720. https://doi.org/10.1056/NEJMoa2002032

[3] Younger, D.S. (2016) I-Cubed (Infection, Immunity, and Inflammation) and the Human Microbiome. Neurologic Clinics, 34, 863-874. https://doi.org/10.1016/j.ncl.2016.05.006

[4] Biot, C., Daher, W., Chavain, N., et al. (2006) Design and Synthesis of Hydroxyferroquine Derivatives with Antimalarial and Antiviral Activities. Journal of Medicinal Chemistry, 49, 2845-2849. https://doi.org/10.1021/jm0601856

[5] Gautret, P., Lagier, J.C., Parola, P., et al. (2020) Hydroxychloroquine and Azithromycin as a Treatment of COVID-19: Results of an Open-Label Non-Randomized Clinical Trial. International Journal of Antimicrobial Agents, 2020, Article ID: 105949.

[6] Geleris, J., Sun, Y., Platt, J., et al. (2020) Observational Study of Hydroxychloroquine in Hospitalized Patients with Covid-19. The New England Journal of Medicine. https://doi.org/10.1056/NEJMoa2012410

[7] Grein, J., Ohmagari, N., Shin, D., et al. (2020) Compassionate Use of Remdesivir for Patients with Severe Covid-19. The New England Journal of Medicine.

[8] Shen, C., Wang, Z., Zhao, F., et al. (2020) Treatment of 5 Critically Ill Patients with 
COVID-19 with Convalescent Plasma. JAMA, 323, 1582-1589.

https://doi.org/10.1001/jama.2020.4783

[9] Cao, W., Liu, X., Bai, T., et al. (2020) High-Dose Intravenous Immunoglobulin as a Therapeutic Option for Deteriorating Patients with Coronavirus Disease 2019. Open Forum Infectious Diseases, 7, ofaa102. https://doi.org/10.1093/ofid/ofaa102

[10] Harahsheh, A.S., Dahdah, N., Newburger, J.W., et al. (2020) Missed or Delayed Diagnosis of Kawasaki Disease during the 2019 Novel Coronavirus Disease (COVID-19) Pandemic. The Journal of Pediatrics. https://doi.org/10.1016/j.jpeds.2020.04.052

[11] Younger, D.S. (2020) Post-Infectious Sequela of SARS-COV-2 Infection in Adults and Children: An Overview of Available Agents and Clinical Responsiveness. Arch Neurology and Neurological Disorder, 3, e102. 